\title{
GERENCIAMENTO DE TECNOLOGIA EM TERAPIA INTENSIVA*
}

\author{
Cátia Romano Madureira** \\ Kátia Veiga** \\ Ana Flávia Mota Sant'ana***
}

\begin{abstract}
MADUREIRA, C.R.; VEIGA, K.; SANT'ANA, A.F.M. Gerenciamento de tecnologia em terapia intensiva. Rev.latinoam.enfermagem, Ribeirão Preto, v. 8, n. 6, p. 68-75, dezembro 2000.
\end{abstract}

\begin{abstract}
A vida do paciente no hospital depende dos procedimentos diagnóstico-terapêuticos e cuidado da equipe de saúde, além da qualidade e disponibilidade de fatores como: estrutura física, recursos materiais, humanos e financeiros. A necessidade de otimizar estes recursos e restaurar a saúde, fez surgir as Unidades de Terapia Intensivas (UTI's), cujo projeto deve considerar o ambiente fisico e equipamentos clínicos indispensáveis ao cuidar. Objetivando identificar o nível de conhecimento dos profissionais de saúde sobre as informações técnico-operacionais contidas nos manuais dos equipamentos e a percepção dos entrevistados quanto a adequação das instalações fisicas e elétricas destas unidades, realizamos um estudo descritivo numa UTI de um hospital público de Salvador. Os resultados mostraram que estas instalações foram consideradas inadequadas ao desempenho seguro de suas atividades, e que a maioria desconhece as especificações técnico-operacionais dos manuais dos equipamentos.
\end{abstract}

UNITERMOS: gerencia, pesquisa em administração de enfermagem, qualidade dos cuidados de saúde

\section{INTRODUÇÃO}

A gestão de serviços de saúde no Brasil, tem-se constituído num grande desafio frente a crise política e econômica que se instalou no setor, o que tem levado à baixa qualidade e produtividade desses serviços.

Diante de tal crise, os gerentes das unidades de saúde deparam-se, com um desafio ainda maior, necessitando articular-se com os demais segmentos da sociedade a fim de obter a utilização racional e eficiente dos recursos que dispõe.

Cabe salientar que num hospital, a vida de um paciente depende não apenas de diagnósticos, exames médicos e cuidados de enfermagem mas, da qualidade e disponibilidade de diversos fatores, como a estrutura física e os recursos materiais, humanos e financeiros.

$\mathrm{O}$ incremento tecnológico vivenciado atualmente, e que teve início após a Segunda Guerra Mundial em todas as áreas do conhecimento, e, em particular na área de saúde, representa um avanço para assistência ao cliente.

Decorrente deste fato, os gerentes defrontam-se com crescentes dificuldades no desenvolvimento da prática da equipe de saúde pela utilização de procedimentos e tratamentos complexos, que exigem uma estrutura física adequada, pessoal capacitado e materiais cada vez mais modernos, pois os níveis de complexidade tecnológica refletem a natureza das tarefas a serem executadas.

A tentativa de otimizar tais recursos e a necessidade de restaurar a saúde e manter a vida, fez surgir as unidades especializadas, entre as quais, as unidades de terapia intensivas (UTI's). Estas, centralizam recursos de apoio às atividades assistenciais no âmbito hospitalar, contribuindo para a melhoria da qualidade dos serviços de saúde, alterando o processo diagnóstico-terapêutico, principalmente no âmbito da prevenção secundária e terciária (DODSON Jr., 1979).

Os projetos físicos de estabelecimentos assistenciais de saúde devem obedecer a Portaria ${ }^{\circ} 1.884 /$ 6M, de novembro de 1994 do Ministério da Saúde, acatando ainda as determinações estabelecidas em códigos, leis e normas relacionadas ao assunto e vigentes no local da construção no âmbito municipal, estadual e

\footnotetext{
* Trabalho apresentado no $50^{\circ}$ Congresso Brasileiro de Enfermagem - Salvador - Bahia - Brasil, set. 1998

** Professoras da Disciplina Administração e Planejamento dos Serviços de Saúde da Escola de Enfermagem da Universidade Federal da Bahia

*** Bolsista PIBIC/CNPQ, aluna do $7^{\circ}$ semestre da Escola de Enfermagem da Universidade Federal da Bahia Endereço - Escola de Enfermagem da Universidade Federal da Bahia - "Campus" Universitario - Vale do Canela - 40110-060 Salvador - Bahia - Brasil
} 
federal, atendendo aos princípios de regionalização, hierarquização, acessibilidade, qualidade e complexidade tecnológica dos serviços de saúde (BRASIL, M.S., 1994).

Assim, nas UTI's, o projeto deverá definir, clara e precisamente, a estrutura física, considerando os ambientes e equipamentos propostos para o uso no setor, e em particular as instalações elétricas.

Desse modo, um projeto físico-funcional para estas unidades deve contemplar todos os componentes, características e materiais a serem utilizados, definindo todos os aspectos dos ambientes necessários ao desenvolvimento das atividades previstas para esta edificação, com vistas à qualidade do cuidado para pacientes e prestadores de serviços, sendo isto também, de responsabilidade dos gerentes desses serviços.

Considerando a segurança no cuidado aos pacientes de unidades especializadas, bem como dos próprios profissionais de saúde atuantes nestes setores, definimos os seguintes objetivos do estudo:

- identificar a percepção dos entrevistados, quanto à adequação das instalações físicas e elétricas das unidades em que desenvolvem suas atividades profissionais;

- avaliar o conhecimento dos profissionais de saúde sobre as informações técnico-operacionais contidas nos manuais dos equipamentos de suporte à vida;

- verificar a atuação do gerente frente às dificuldades identificadas pelos profissionais de saúde contribuindo para a manipulação segura dos equipamentos de suporte à saúde.

\section{REVISÃO DE LITERATURA}

Sabe-se que a eletricidade permeia, na sociedade moderna, todas as formas de atividade do homem. Habituado ao seu uso, muitas vezes, este, como refere DYTZ (1983), desconhece e desconsidera os perigos associados ao seu emprego. Sofre, em conseqüência, danos corporais provocados por problemas, como o choque elétrico.

O homem considerado "saudável" pode sofrer sérios danos provenientes do choque elétrico. Piores ainda, são as alterações que pode causar, em se tratando de um paciente internado nas Unidades Especializadas. Esse já se encontra vulnerável, devido às suas próprias condições físicas, além de, na maioria das vezes, ficar totalmente exposto a uma quantidade enorme de equipamentos clínicos, dependendo, portanto, da utilização correta dos mesmos pela equipe de saúde.

Os pacientes e profissionais de saúde também podem estar expostos a acidentes elétricos, cujo maior perigo consiste no "vazamento" de corrente elétrica, ou corrente de fuga, a qual pode passar do monitor para o paciente, quando o corpo integra um circuito elétrico, principalmente quando mais de um equipamento está sendo usado, como informa MELTZER et al. (1984).

O corpo integra um circuito elétrico quando está entre dois pontos de potenciais diferentes. A corrente elétrica entra, portanto, no corpo por um ponto e sai em outro ponto (WEBSTER, 1978).

Salientam-se alguns danos corporais causados pela passagem da corrente elétrica pelo corpo, como queimaduras eletrotérmicas ou elétricas, que, segundo FORTES (1986), podem produzir um quadro grave, porque a eletricidade afeta, em graus variáveis, todos os tecidos por onde passa, e a fibrilação ventricular, que ocorre mais facilmente quando a intensidade e a densidade da corrente elétrica, ao nível do coração, são mais elevados. OLIVEIRA Jr. et al. (1982) e JAMES et al. (1990) consideram que a fibrilação ventricular ocorre, provavelmente, quando o sistema de condução, encontrase danificado.

Além destes, podem ser citados as alterações vasculares, extra-sístoles ventriculares, a inversão da onda T e o infarto do miocárdio, uma vez que a corrente elétrica pode atuar diretamente sobre o miocárdio ou sobre as coronárias, ocasionando um espasmo destas últimas. As alterações ósseas ocorrem porque o osso oferece uma resistência elétrica maior do que qualquer tecido, ocorrendo, portanto, um super aquecimento quando conduzindo uma corrente elétrica (THOMPKINS et al., 1990).

Desse modo, é de responsabilidade da gerência dessas unidades, observar os fatores relacionados à segurança elétrica.

Assim, os dispositivos de aterramento devem obedecer a seguinte especificação do Ministério da Saúde:

"a uma distância nunca superior a 3,00 m

de cada leito, deverá ser previsto um ponto de aterramento, contendo conectores para o aterramento de todas as tomadas e superfícies condutoras".

Qualquer superfície que poderá se tornar eletricamente energizada, com exceção de pequenos objetos, deverá ser ligada ao terra do paciente, compondo o que WEBSTER (1978) chama de aterramento equipotencial.

Segundo STANLEY(1981), nas instalações em que o sistema de aterramento é projetado e calculado previamente, é interessante fazer a medição da resistência de "terra" após a execução da obra no campo, onde devem ser observados alguns parâmetros como a resistividade do solo, a profundidade dos eletrodos de aterramento, dimensões dos eletrodos e a qualidade do material de que são feitos os eletrodos.

Quanto à manutenção de equipamentos, o grupo de trabalho do SEPLAN/CNPq (1985) cita estudos em 
quatro instituições nos Estados Unidos, onde dispensase grande importância, à manutenção e reparo, e aos níveis de corrente de fuga dos equipamentos hospitalares. Estes níveis podem causar perigo, tanto para os operadores dos equipamentos, quanto para os pacientes que os utilizam. Estas instituições utilizam instrumentos de teste que captam, amplificam e exibem a corrente da fuga.

Considerando estes estudos, torna-se necessário o estabelecimento de políticas de manutenção nas instituições hospitalares, enfocando a manutenção preventiva, a qual, além de aumentar a longevidade dos equipamentos, garante a segurança elétrica do paciente e operadores e conseqüentemente, promove uma assistência de qualidade.

No que se refere à recursos humanos, estes devem estar constantemente atualizados, devido aos avanços tecnológicos que vem ocorrendo de forma quase que exponencial no setor saúde. Entende-se por tecnologia, um conjunto de conhecimentos aplicados a uma determinada área, indo desde equipamentos de alto grau de complexidade, até procedimentos considerados mais simples, como por exemplo uma punção venosa ou mesmo um banho no leito. Para tanto, há permanente necessidade de capacitação de pessoal, sendo prevista como estratégia institucional a otimização de tempo e produtividade destes recursos.

Cabe à organização, no caso, os hospitais, comungar esforços no sentido de recrutar e selecionar profissionais do mercado de trabalho, ambientá-los, desenvolvê-los e incorporá-los ao espaço produtivo, empregando-os para atender as necessidades da organização e do profissional. Aos gerentes, cabe criar espaços de inovação e cooperação, pois os seres humanos devem ser entendidos como seres ativos e dinâmicos, garantindo ainda, a sua empregabilidade num mercado altamente competitivo pela atual filosofia de globalização da economia mundial (CHIAVENATO,1992).

Desse modo, a estrutura físico-funcional nesses setores, deve favorecer a manipulação segura dos equipamentos utilizados para dar suporte à vida dos pacientes, considerando as normas de operação destes, tanto em relação ao próprio paciente, quanto ao profissional de saúde, que os adota como ferramenta indispensável para o desenvolvimento de suas atividades assistenciais.

Assim, é de responsabilidade dos gerentes prever e prover recursos humanos e materiais adequados à realidade deste processo de cuidar.

\section{METODOLOGIA}

Realizamos um estudo descritivo, numa UTI de um hospital público, federal, geral e de grande porte da cidade de Salvador-BA, considerado como unidade de referência do Estado.

Utilizamos para coleta de dados um questionário com questões fechadas dirigido aos profissionais da equipe de saúde da unidade de terapia intensiva do referido hospital, aplicados nos três turnos de funcionamento: manhã, tarde e noite, totalizando 57 respondentes na nossa população, constituída por 16 enfermeiros, 27 auxiliares de enfermagem e 14 médicos.

O instrumento (em anexo) foi aplicado no período de novembro de 1997 à abril de 1998, após a apreciação e o consentimento da instituição em apreço. Os dados coletados foram processados pelo programa EPINFO.

\section{RESULTADOS}

Como podemos observar na Figura 1, dos 57 profissionais da equipe de saúde entrevistados, 78,95\% (45) eram do sexo feminino, com predominância da faixa etária entre 25 a 30 anos.

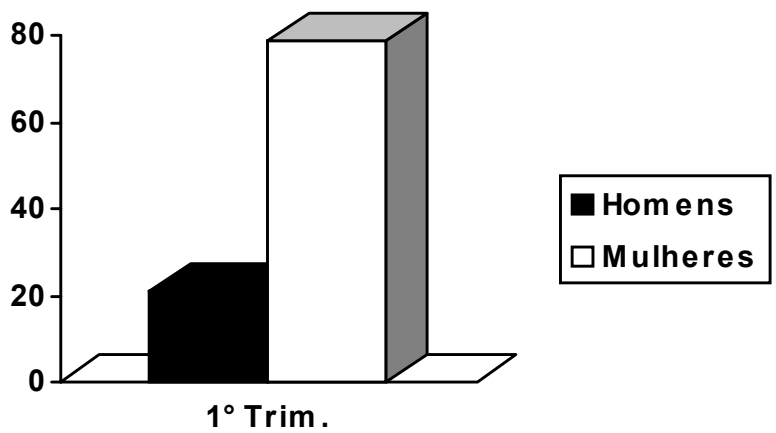

Figura 1 - Distribuição dos profissionais de saúde por sexo. Salvador, 1998

Dos respondentes, 47,37\% (27) eram auxiliares de enfermagem, 24,56\% (14) médicos, seguidos de $28,07 \%$ (16) de enfermeiros, conforme verificamos na figura 2.

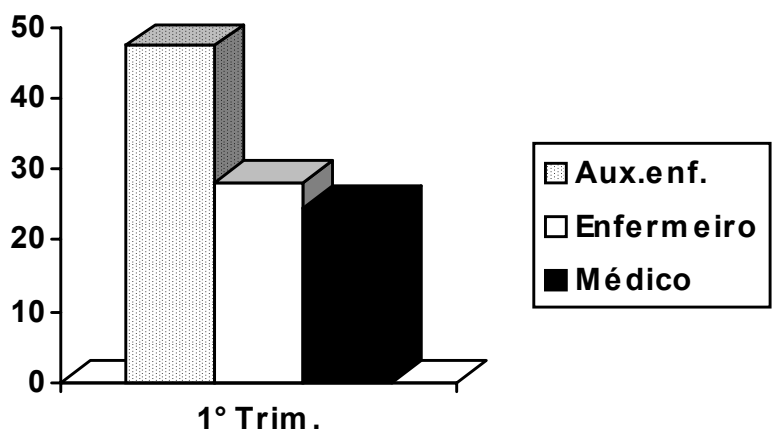

Figura 2 - Distribuição dos profissionais de saúde por categoria profissional. Salvador, 1998 
Quanto ao tempo de serviço em UTI, 73,06\% (42) dos entrevistados tinham até 06 anos de serviço, sendo que 78,95\% (45) dos respondentes informaram participação em treinamentos específicos nesta área.

Analisando os dados acima, observamos que a UTI tem um quadro de pessoal jovem, com pouco tempo de serviço nesta especialidade. Considerando a dinâmica do setor, onde há exigência de intenso trabalho físico e mental, além do desgaste emocional, pois lidam com situações que envolvem riscos iminentes de vida, acreditamos que se o emprego de mão de obra mais experiente implica em pessoal com uma maior destreza e habilidade, além de discernimento ao tomar decisões importantes para a manutenção da vida do paciente sobre a nossa responsabilidade, o emprego de pessoal mais jovem, provavelmente se traduz em maior energia e disposição para o trabalho.

A equipe de saúde é formada predominantemente pela equipe de enfermagem, com significante percentual de auxiliares de enfermagem, o que determina a prevalência do sexo feminino. Este fato está de acordo com dados divulgados pelo COREN-BA**** (1998), que mostra a enfermagem como sendo o maior contingente de trabalhadores de saúde e ainda, com alto percentual de auxiliares de enfermagem dentro do quadro funcional da enfermagem. Estes dados apontam para um número pouco expressivo de técnicos de enfermagem no Estado da Bahia.

Considerando ser esta unidade especializada, defendemos a necessidade de pessoal capacitado, representado por enfermeiros e técnicos de enfermagem, o que encontra respaldo na Resolução COFEn-183, que dispõe sobre o dimensionamento de pessoal para o serviço de enfermagem. Entretanto, apesar destas unidades lidarem com o que há de mais sofisticado em termos de tecncologia de suporte à vida, ainda observamos o emprego de um maior número de auxiliares de enfermagem, categoria de menor formação dentro da equipe de enfermagem, contrapondo-se aos dados da literatura e legislação profissional vigente.

Os enfermeiros entrevistados, informaram lidar mais diretamente com atividades voltadas ao gerenciamento destas unidades, delegando a assistência aos demais membros da equipe de enfermagem, não sendo assim considerados o grau de complexidade exigido no cuidado aos pacientes sob sua responsabilidade.

Quanto às instalações físicas, 82,46\% ( 47 ) dos entrevistados consideraram as instalações físicas da UTI inadequadas ao desenvolvimento dos trabalhos nesta unidade, (Figura 3).

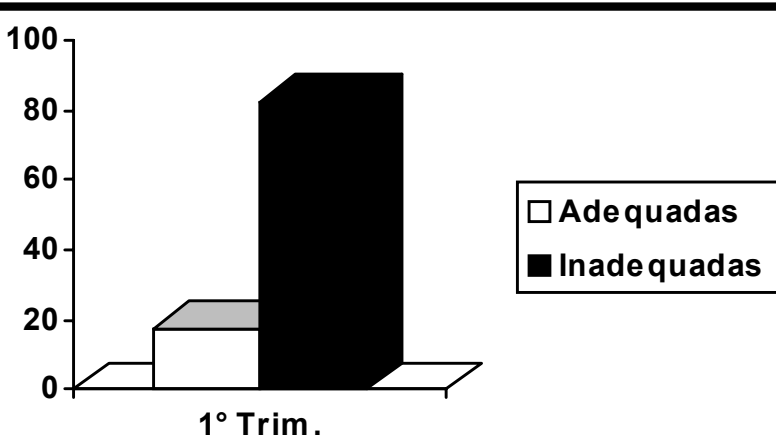

Figura 3 - Adequação das instalações físicas às atividades desenvolvidas na UTI: opinião dos profissionais de saúde.Salvador, 1998

Estes dados refletem um cenário preocupante, uma vez que este serviço é considerado de referência no Estado da Bahia. Salientamos que a estrutura física deve proporcionar um ambiente de segurança e bem-estar para os profissionais e clientes, facilitando o desenvolvimento das atividades assistenciais, com redução de esforços físico, mental e conseqüentemente o "stress". Desta forma alcança-se uma assistência de excelência, considerada objetivo maior do processo de gerenciamento.

No que se refere aos equipamentos, os entrevistados, de um modo geral, informaram que estes facilitam o desenvolvimento de suas atividades, reconhecendo a grande dependência entre a prática por ele exercida e os equipamentos clínicos adotados.

$O$ processo de cuidar nas unidades especializadas depende grandemente do uso racional e seguro de equipamentos de suporte à vida, entre outros fatores. Sendo assim, cabe aos gerentes desses setores a responsabilidade de considerar os riscos provenientes, principalmente dos danos elétricos a que podem estar expostos, tanto o paciente, quanto os profissionais de saúde.

Sabe-se entretanto que, para garantir a qualidade do cuidar com vistas à longevidade dos equipamentos e a manutenção da segurança elétrica para os profissionais e pacientes, faz-se necessário uma política de manutenção efetiva. Porém, encontramos no nosso estudo a inexistência de um programa de manutenção sistemático, sendo esta requerida apenas para o conserto dos equipamentos, quando ocorrem falhas técnicooperacionais.

Quanto ao grau de conhecimento dos profissionais na área de saúde destas unidades, em relação as especificações técnicas contidas nos manuais de operação dos equipamentos, encontramos uma realidade distante de um contexto de segurança, vez que $38,9 \%$ 
(22) dos entrevistados informaram desconhecimento do mesmo. Tal cenário evidencia uma grave situação quando, se por um lado, 59,5\% (34) informaram ter participado de algum treinamento formal específico com relação ao manuseio adequado dos equipamentos, por outro, disseram não interagir efetivamente com os técnicos de manutenção. Diante do exposto, questionamos a viabilidade e a direcionalidade deste treinamento, visto o depoimento de alguns entrevistados que verbalizaram quando da leitura do questionário: "o que é manual"?

Entendemos ser imprescindível o conhecimento das normas técnico-operacionais dos equipamentos pelos profissionais de saúde uma vez que informaram ser, os equipamentos, ferramentas importantes no seu processo de trabalho.

MADUREIRA (1993) salienta a importância da promoção de treinamento e/ou reciclagem pela instituição, a fim de que os profissionais possam conhecer os princípios de segurança elétrica que envolvem profissionais de saúde e pacientes, sendo tal providência de responsabilidade dos gerentes. Tal medida, acarretará melhor controle por parte destes, no que se refere à manutenção preventiva e corretiva dos equipamentos, bem como o uso adequado. Ocorrerá, também, a fiscalização mais rigorosa da manutenção nas instalações elétricas das unidades especializadas, que poderá ser efetuada pelos técnicos do próprio hospital.

Tais medidas ocasionarão a redução dos custos hospitalares, além de uma assistência de melhor qualidade aos clientes, gerando a conseqüente satisfação dos mesmos e de toda a equipe de saúde.

Quanto aos recursos humanos, 45,46\% (26) dos informantes da UTI consideraram haver adequação e suficiência quanti-qualitativa destes para atender às necessidades da unidade.

O estabelecimento de um quantitativo de pessoal capaz de proporcionar boa qualidade de assistência à saúde, também é responsabilidade do gerente da unidade. Para tanto, faz-se necessário uma análise criteriosa da situação, a fim de ser determinado o parâmetro que melhor se adeqüe à realidade do serviços.

Vale ressaltar que esta questão é bastante polêmica, como demonstram os estudos realizados por SANTOS (1992), nos quais os resultados da aplicação de várias fórmulas para dimensionamento de pessoal de enfermagem, denotam uma estreita variação entre os métodos padronizados pelos vários autores consultados por esta autora.

O preparo dessa mão de obra envolve, basicamente, treinamento de pessoal entendido como um processo de mudança planejada, que visa a obtenção de determinado comportamento com o mínimo de esforço e o máximo de rendimento e satisfação para o profissional e a instituição.
Este treinamento deve ser visto como um processo político de desenvolvimento de pessoal (MARCONDES, 1978). A meta, portanto, deve atingir os objetivos organizacionais e profissionais, devendo constituir inclusive, parte do processo seletivo (MARTINS, 1983).

Assim, defendemos a importância da utilização dos manuais de operação para os usuários e os manuais de instalação, calibração e manutenção por parte da equipe de manutenção dos hospitais, como parte do processo de treinamento destes funcionários, a fim de que haja uma otimização do uso dos equipamentos clínicos, segurança na manipulação, redução de custos para a instituição, além de garantir uma assistência ininterrupta e conseqüentemente, um produto de qualidade.

Considerando que a padronização é uma das ferramentas imprescindíveis ao programa de qualidade, uma vez que possibilita a reunião de resultados através de instrumentos de controle e avaliação, não podemos avaliar, portanto, a qualidade no processo de gerenciamento sem comentarmos a aplicação destes instrumentos na instituição.

Na UTI 63,16\% (36) dos respondentes referem conhecer os critérios de garantia da qualidade utilizados nos equipamentos de suporte a vida. Entretanto, apenas $19,3 \%$ (11) conhecem em sua totalidade os instrumentos utilizados no controle/avaliação destes equipamentos.

Sabe-se que o controle/avaliação consiste, basicamente, na observação e comparação do desempenho efetivo dos equipamentos em relação ao estabelecido, considerando-se padrões de quantidade e qualidade, o que se reflete no controle de desvios das operações em andamento, nos resultados finais e na qualidade da assistência. (ARNDT \& HUCKABAY, 1983).

A unidade especializada estudada vale-se de um aparato tecnológico complexo e variado, para auxiliar a monitorização e sustentação da vida, representando conseqüentemente, um avanço no sentido da assistência ao cliente. Ao mesmo tempo, tal avanço dificulta a proteção da equipe de saúde, e principalmente a de enfermagem, por defrontar-se com materiais por vezes desconhecidos, e cada vez mais sofisticados (ROMANO \& VEIGA, 1998).

Entretanto, na estrutura hospitalar, muitas vezes, a vida dos profissionais é colocada em risco pela falta de condições de exercer o trabalho. Isso provavelmente ocorre porque o objetivo e a filosofia do hospital de garantir a qualidade da assistência parece ser negligenciado quando se observa a prevalência de propostas para diminuição dos custos, em detrimento do bom atendimento ao usuário.

Assim sendo, acreditamos serem os gerentes de 
serviços na área de saúde atores importantes para o desenvolvimento da assistência segura e de qualidade à clientela, para o que é indispensável o uso racional e otimizado dos equipamentos de suporte a vida.

\section{CONSIDERAÇÕES FINAIS}

O processo de gerenciamento visa, entre outros aspectos, a otimização dos recursos, aumentando, conseqüentemente, a produção dos serviços, reduzindo custos e possibilitando a eficiência e eficácia dos procedimentos, o que resulta em qualidade da assistência. Assim sendo, faz-se necessário a integração entre os profissionais da equipe de saúde para que estes atuem, concretamente, de acordo com a filosofia e objetivos da instituição.

O compromisso dos profissionais da equipe de saúde no gerenciamento de recursos que viabilizam o processo de cuidar é indispensável para uma assistência isenta de riscos, e o uso racional dos materiais e equipamentos indispensáveis ao desenvolvimento das atividades envolvidas neste processo.

Entretanto neste estudo, encontramos um cenário preocupante, onde as instalações físicas e elétricas, foram consideradas inadequadas ao desenvolvimento efetivo e seguro das atividades dos profissionais de saúde, e estes informaram o desconhecimento sobre as informações contidas nos manuais de operação dos equipamentos de suporte à vida, considerados por eles ferramentas imprescindíveis no processo de cuidar.
Com base na análise destes dados, podemos inferir que a atuação dos gerentes dos serviços em apreço, estava limitada ao cumprimento das rotinas do serviço atendendo às expectativas da cúpula administrativa da instituição, em detrimento das reais dificuldades dos serviços, haja visto que o treinamento de pessoal não atendia às necessidades de manuseio seguro e adequado dos equipamentos de suporte a vida.

Acreditamos que a atuação dos gerentes dos serviços de saúde devem contemplar, entre outros fatores: - a previsão e o controle dos recursos necessários a prestação do cuidado aos pacientes sob a sua responsabilidade, capacitando os RH para a manipulação segura e eficiente dos materiais e, sobretudo, dos equipamentos de suporte a vida;

- a adequação das instalações físicas às atividades que serão desenvolvidas, favorecendo assim a manutenção de um ambiente terapêutico, agradável e seguro para os profissionais e pacientes;

- a proteção, conservação e manutenção dos materiais e em particular, dos equipamentos imprescindíveis ao cuidar, contribuindo desse modo, para a longevidade dos mesmos; e,

- implantar um sistema de registro das informações a cerca dos recursos, favorecendo assim, o controle do Processo de Trabalho.

Através da adoção destas medidas, cremos ser possível a avaliação da qualidade do serviço, sendo esta um dos indicadores do grau de excelência do cuidado prestado por estas instituições.

\section{MANAGEMENT OF TECHNOLOGY IN INTENSIVE CARE UNITS}

The lives of hospitalized patients depend on diagnostic-therapeutic procedures and on the care provided by the health team. It also depends on the quality and availability of factors such as: physical structure and material and human and financial resources. The need to optimize these resources and re-establish patients' health has brought about the Intensive Care Units (ICU), whose design must consider the physical environment and clinical equipment which are indispensable in health care. Aiming at identifying the level of knowledge of health professionals as to technical and operational information in equipment manuals and the perception of the interviewed professionals in relation to the adequacy of the physical and electrical installations of such units, we conducted a descriptive study in an ICU of a public hospital in Salvador, Brazil. The results show that such installations were considered to be unsuitable for the safe development of activities and that most of the health team members did not know the technical or operational specifications in the equipment manuals.

KEY-WORDS: management, research in nursing administration, health care quality

\section{GERENCIAMIENTO DE TECNOLOGÍA EN TERAPIA INTENSIVA}

La vida del enfermo en el hospital depende de los procedimientos diagnóstico-terapéuticos y del esmero del equipo de salud, además de la calidad y disponibilidad de factores como: organismo físico, recursos materiales, humanos y financieros. La necesidad de mejorar estos recursos y restaurar la salud, hizo surgir las Unidade de Terapia Intensiva (UTIS) cuyo proyecto debe considerar el ambiente físico y los equipos clínicos indispensables para el cuidado. Con el objeto de identificar los conocimientos de los profesionales de salud sobre las informaciones técnico-operacionales existentes en los manuales de los equipos y la 
percepción de los entrevistados en relación con la adecuación de las instalaciones físicas y eléctricas (de estas unidades), hicimos un análisis en una UTI de un hospital general de Salvador. Los resultados mostraron que estas unidades son inseguras para las actividades y que la mayor parte de las personas desconoce las especificaciones técnico-operacionales de los manuales de los equipos.

TÉRMINOS CLAVES: gerencia, investigación de administración en enfermería, calidad de cuidados de salud

\section{ANEXO 1}

UNIVERSIDADE FEDERAL DA BAHIA - ESCOLA DE ENFERMAGEM

DEPARTAMENTO DE ENFERMAGEM MÉDICO-CIRÚRGICA - DEMACAE

GRUPO DE ESTUDOS E PESQUISA EM ADMINISTRAÇÃO DE SERVIÇOS DE ENFERMAGEM - GEPASE

PROJETO INTEGRADO DE PESQUISA - GERENCIAMENTO E QUALIDADE DA ASSISTENCIA EM UNIDADES DE TERAPIA INTENSIVA E EMERGENCIA DE HOSPITAIS GERAIS EM SALVADOR/BAHIA

\section{QUESTIONÁRIO}

Hoje, mais do que nunca, faz-se necessário avaliar as características gerenciais nas Unidades de Tratamento Intensivo e Emergência para que possam atender com segurança os clientes internos e externos. Para tanto, solicitamos a sua colaboração no sentido de responder as questões abaixo de acordo com o seu ponto de vista, assinalando com um "X" apenas uma alternativa. Não escreva o seu nome. Desde já, agradecemos a sua valiosa participação.

\section{IDENTIFICAÇÃO}

\begin{tabular}{|l|l|}
\hline Sexo: ( ) M ( ) F & Tempo de serviço: \\
\hline Idade: & Tempo de serviço no setor: \\
\hline *Profissão: & $\begin{array}{l}\text { Participou de treinamento específico nesta unidade: } \\
\text { ( ) Sim ( ) Não }\end{array}$ \\
\hline
\end{tabular}

* Quando for gerente ou chefe informar o exercício da função

\section{QUESTÕES}

\begin{tabular}{|c|c|c|c|c|}
\hline VARIÁVEIS & QUESTÕES & DISCORDO & $\begin{array}{l}\text { NÃO CONCORDO } \\
\text { NEM DISCORDO }\end{array}$ & CONCORDO \\
\hline \multirow[t]{4}{*}{ ESTRUTURA } & $\begin{array}{l}\text { As instalações físicas desta unidade são adequadas ao desenvolvimento do } \\
\text { seu trabalho. }\end{array}$ & & & \\
\hline & Os recursos humanos desta unidade são quantitativamente e suficientes. & & & \\
\hline & Os recursos humanos desta unidade são qualitativamente adequados. & & & \\
\hline & Os recursos materiais desta unidade são adequados e suficientes. & & & \\
\hline \multirow[t]{5}{*}{ EQUIPAMENTOS } & Os equipamentos desta unidade facilitam o seu trabalho. & & & \\
\hline & $\begin{array}{l}\text { Existe cooperação entre a equipe desta unidade e a equipe de manutenção } \\
\text { dos equipamentos. }\end{array}$ & & & \\
\hline & $\begin{array}{l}\text { A manutenção elétrico-eletrônica dos equipamentos desta unidade é feita } \\
\text { periodicamente. }\end{array}$ & & & \\
\hline & É grande a dependência do seu trabalho com relação aos equipamentos. & & & \\
\hline & $\begin{array}{l}\text { É suficiente o seu grau de conhecimento em relação às especificações contidas } \\
\text { nos manuais de operações dos equipamentos. }\end{array}$ & & & \\
\hline \multirow[t]{3}{*}{ CONTROLE } & $\begin{array}{l}\text { Os instrumentos de controle e avaliação utilizados pelo serviço são amplamente } \\
\text { conhecido. }\end{array}$ & & & \\
\hline & $\begin{array}{l}\text { Os instrumentos de controle e avaliação utilizados pelo serviço são } \\
\text { compartilhados pelos membros da equipe. }\end{array}$ & & & \\
\hline & $\begin{array}{l}\text { Os critérios de garantia de qualidade desta unidade são de conhecimento de } \\
\text { todos os membros da equipe. }\end{array}$ & & & \\
\hline \multirow{7}{*}{$\begin{array}{l}\text { ATUAÇÃO DO } \\
\text { GERENTE }\end{array}$} & Os gerentes deste setor cumprem agenda de reuniões administrativas. & & & \\
\hline & & & & \\
\hline & O gerente desta unidade atua como facilitadora do trabalho da equipe. & & & \\
\hline & O gerente aceita as sugestões dos subordinados. & & & \\
\hline & $\begin{array}{l}\text { O gerente demonstra comprometimento com a qualidade da assistência desta } \\
\text { unidade. }\end{array}$ & & & \\
\hline & $\begin{array}{l}\text { O gerente demonstra compromisso com o desenvolvimento dos seus } \\
\text { subordinados. }\end{array}$ & & & \\
\hline & O gerente demonstra preocupação com a saúde dos seus subordinados. & & & \\
\hline
\end{tabular}




\section{REFERÊNCIAS BIBLIOGRÁFICAS}

01. ARNDT, C.; HUCKABAY, L.M.D. Administração em Enfermagem. Rio de Janeiro: Interamericana, 1983.

02. BRASIL. Ministério da Saúde. Secretária de Assistência à Saúde. Departamento de Normas Técnicas. Normas para projetos físicos de estabelecimentos assistenciais de saúde. Brasília, 1994. p.11-26.

03. BAHIA. Conselho Regional de Enfermagem. Boletim Informativo, v. 14, n. 23, mar/abr/mai. 1998.

04. CHIAVENATO, I. Gerenciando pessoas. São Paulo: Makron Books, 1992.

05. DODSON Jr., B. Facilities in clinical engineering: principles and pratices. Englewood Cliffs: Pratice - Hall, 1979.

06. DYTZ, E. Aspectos da segurança elétrica em hospitais. Rio de Janeiro, 1983. Dissertação (Mestrado) - COPPE, Universidade Federal do Rio de Janeiro.

07. FORTES, J.I. Enfermagem em emergências. São Paulo: EPU, 1986. p. 61.

08. JAMES, T.N. et al. Cardiac abnormalities demonstrated postmortem in four cases of accidental eletrocution and their potential significance relative to nonfatal eletrical injuries of the heart. Am.Heart.J., v. 120, n. 1, p. 143$157,1990$.

09. MADUREIRA, C.M.R. O enfermeiro e os equipamentos clínicos: estudo em um Centro de Terapia Intensiva. Rio de Janeiro, 1993.188p. Dissertação (Mestrado) - Escola de Enfermagem Anna Nery, Universidade Federal do Rio de Janeiro.
10. MARCONDES, R.S. Treinamento de pessoal em hospitais. Rev. Paul. Hosp., v. 26, n. 10, p. 436, 1978.

11. MARTINS, M.L.R. O Serviço de Enfermagem: administração e organização. São Paulo: CEDAS, 1983.

12. MELTZER, L.E. et al. Enfermagem na Unidade Coronariana. Rio de Janeiro/São Paulo: Atheneu, 1984. p. 149.

13. OLIVEIRA Jr., W. et al. Infarto do miocárdio após choque elétrico: apresentação de um caso. Arq. Bras. Cardiol., v. 39, n. 5, p. 325-327, nov. 1982.

14. ROMANO, C.; VEIGA, K. Atuação da enfermagem no gerenciamento de recursos materiais em Unidades de Terapia Intensiva (UTI's). Rev.Bras.Enfermagem, v. 51, n. 3, p. 485-492, jul./set. 1998.

15. Santos, S. Cálculo de pessoal de Enfermagem : estudo de dois métodos. Rev. Esc. Enfermagem USP, v. 26, n. 2, p. 137-154, ago. 1992.

16. SEPLAN/CNPq. A instrumentação biomédica e o programa da engenharia de manutenção nos hospitais brasileiros. Brasília, 1985.

17. STANLEY, P.E. CRC Handbook of hospital safety. Flórida: CRC-PRESS, 1981.

18. THOMPKINS, G.S. et al. Bilateral simulataneous fratures of the femoral neck: case report. J.Trauma, v. 30, n. 11, p. 1415, nov. 1990.

19. WEBSTER, J.G. Medical instrumentation: aplication and design. Boston: editora, 1978.

Recebido em: 8.1.1999 